\title{
Um olhar para as experiências de Alimentação e Nutrição do Prêmio APS Forte
}

\author{
Ana Carolina Lucena Pires, Mayara Kelly Pereira Ramos, Maria Fernanda Moratori Alves, \\ Rafael Dall Alba, Gisele Ane Bortolini, Juliana Rezende Melo da Silva
}

RESUMO

O aprimoramento da agenda de alimentação e nutrição (A\&N) é essencial para fortalecer os atributos da Atenção Primária à Saúde (APS). Este trabalho analisa as experiências de A\&N inscritas no Prêmio APS Forte, apontando potencialidades, desafios e perspectivas futuras. Foram avaliadas 40 experiências, que foram categorizadas quanto às diretrizes da PNAN e aos atributos da APS. A maioria das ações ocorreu nas unidades de saúde com foco no sobrepeso/obesidade por meio de abordagem coletiva. As principais diretrizes fortalecidas foram a vigilância alimentar e nutricional (VAN) e a promoção da alimentação adequada e saudável. Predominaram os atributos de orientação comunitária, acesso de 1o contato e longitudinalidade. Percebeu-se que os profissionais reconhecem o problema da obesidade, entretanto, a maioria das intervenções tem como foco apenas ações de educação em saúde, sendo a obesidade um tema que demanda fortalecimento de todos os atributos da APS e articulação intersetorial, principalmente entre saúde, educação, assistência social e agricultura familiar.

Palavras-chave: Atenção Primária à Saúde; Programas e Políticas de Nutrição e Alimentação; Educação Alimentar e Nutricional; Promoção da Saúde; Sistema Único de Saúde.

Revista da Rede APS 2020

Publicada em: 04/09/2020

DOI:10.14295/aps.v2i3.153

Ana Carolina Lucena Pires (DESF/SAPS/MS)

Mayara Kelly Pereira Ramos (DESF/SAPS/MS)

Maria Fernanda Moratori Alves (DESF/SAPS/MS)

Rafael Dall Alba (OPAS/OMS)

Gisele Ane Bortolini (DESF/SAPS/MS)

Juliana Rezende Melo da Silva (DESF/SAPS/MS)

\section{ABSTRACT}

Correspondência para:

The improvement of the food and nutrition (A\&N) commitment is essential to strengthen the attributes of Primary Health Care (PHC). This work analyzes the A\&N experiences registered in the APS Forte Award, pointing out potentialities, challenges and future perspectives. Forty experiences were evaluated, which were categorized according to PNAN guidelines and PHC attributes. Most of the actions took place in health units with a focus on overweight / obesity through a collective approach. The main strengthened guidelines were the promotion of adequate and healthy food and food and nutritional surveillance (FNS) ed that professionals recognize the problem of obesity, however, most interventions focus only on health education actions, with obesity being a theme that demands strengthening of all PHC attributes and intersectoral articulation, especially between health, education, social assistance and family farming.

Keywords: Primary Health Care; Nutrition Programs and Policies; Food and Nutrition Education; Health Promotion; Unified Health System.

Ana Carolina Lucena Pires ana.lucena@saude.gov.br 


\section{INTRODUÇÃO}

A má alimentação lidera o ranking dos fatores de risco relacionados à carga global de doenças no mundo, tendo em vista que as características da dieta não estão relacionadas apenas com o bem estar dos indivíduos, mas também pelo aparecimento de doenças crônicas, como obesidade, hipertensão arterial, diabetes e câncer (Malta et al., 2017; Swinburn et al., 2019). O elevado consumo de sal, a baixa ingestão de grãos integrais e o baixo consumo de frutas foram os principais fatores dietético que impactaram na mortalidade e anos de vida perdidos no mundo (Afshin et al., 2019).

No Brasil, a alimentação e a saúde são direitos constitucionais (Brasil, 1990; Constituição Federal, 2000), por isso, foi definido que compete à direção nacional do Sistema Único de Saúde (SUS) formular, avaliar e apoiar políticas de alimentação e nutrição, cabendo a execução às esferas estadual e municipal (Brasil, 1990). Nesse sentido, sob o propósito de melhorar as condições de saúde e nutrição da população brasileira, por meio da vigilância alimentar e nutricional, do estímulo à alimentação adequada e saudável e prevenção e controle de agravos nutricionais, instituiu-se a Política Nacional de Alimentação e Nutrição (PNAN), que completou 20 anos em 2019 (Brasil, 1999, 2012).

Nas últimas décadas, a melhoria das condições de vida dos brasileiros reduziu o contexto de desnutrição e deficiências nutricionais de principal expressão de insegurança alimentar e nutricional à uma condição persistente apenas em grupos populacionais específicos (Brasil, 2012, 2014; Lima, de et al., 2010; Monteiro et al., 2009). Concomitantemente, houve um rápido processo de transição nutricional, acompanhado por alterações no padrão alimentar da população, que vem substituindo o consumo de alimentos básicos por ultraprocessados, levando ao aumento expressivo do sobrepeso e da obesidade em todas as faixas etárias (Brasil, 2010, 2020).

Atualmente, o sobrepeso e a obesidade são as condições mais prevalentes na população brasileira resultantes da alimentação inadequada (Brasil, 2010, 2020). O excesso de peso acomete mais da metade da população adulta (56,9\%), representando $80 \%$ dos indivíduos diabéticos e hipertensos (Nilson et al., 2020), e uma em cada três crianças brasileiras (Brasil, 2010, 2020), sendo responsável pelo aumento da ocorrência de uma série de outras doenças crônicas, como hipertensão, diabetes e câncer, que oneram o SUS (Nilson et al., 2020; Tremmel et al., 2017). Sabe-se que a obesidade pode ser prevenida (Carvalho et al., 2013; Dabas e Seth, 2018; German, 2016; Langer, 2018) e por consequência, reduzir o número de indivíduos com doenças crônicas, que são responsáveis por $72 \%$ das mortes no país (Malta et al., 2014).

A Atenção Primária à Saúde (APS) tem como objetivo a melhoria da saúde individual e populacional, com equidade, sendo orientada por atributos essenciais e derivados, que quanto mais presentes, potencializam a qualidade da atenção (Starfield, 2002). Embora sejam muitos os avanços observados na última década, estudos e iniciativas de avaliação da qualidade da atenção proporcionada pelas equipes de Saúde da Família no SUS apontam que o acesso é o atributo da APS que mais precisa ser fortalecido no país (Araújo, de et al., 2014; Castro, de et al., 2012; Facchini, Tomasi e Dilélio, 2018; Kemper, 2019).

De forma a contribuir com o aprimoramento da agenda de alimentação e nutrição na APS, o objetivo do presente trabalho é realizar a análise das experiências inscritas no Prêmio APS Forte que trataram sobre esta temática, apontando potencialidades, desafios e perspectivas futuras.

\section{METODOLOGIA}

O presente trabalho é um estudo descritivo que usou como base os relatos de experiência classificados na categoria alimentação e nutrição submetidos ao Prêmio APS Forte para o SUS 2019. O prêmio foi uma iniciativa do Ministério da Saúde juntamente com a Organização Pan-americana de Saúde (OPAS) para identificar experiências locais, municipais 
ou regionais que promovam a melhoria do acesso da população (tema central desta edição), priorizando e reforçando o papel da APS como porta de entrada prioritária e coordenadora da atenção no sistema de saúde.

A iniciativa recebeu 1.294 inscrições de experiências na APS provenientes de todos os Estados do país e o Distrito Federal. A maior parte das inscrições foi proveniente de equipes de Saúde da Família (533), seguida por representantes de Secretarias Municipais de Saúde (247) e outros tipos (183), como inscrições individuais e de outros equipamentos públicos, como UBS, escolas e unidades prisionais. Dentre estas, 41 experiências foram classificadas na temática de Alimentação e Nutrição (AN), as quais são objeto de estudo desse artigo. Porém, foi excluído um relato por não se tratar de experiência desenvolvida no âmbito da APS e outro, por estar duplicado no banco de dados, totalizando 39 trabalhos considerados.

Todas as experiências recebidas foram consolidadas em uma planilha de excel e analisadas por 4 avaliadores (nutricionistas) da Coordenação Geral de Alimentação e Nutrição do Ministério da Saúde. Os relatos foram categorizados quanto às diretrizes da PNAN e aos atributos essenciais e derivados da Atenção Primária à Saúde (APS). São diretrizes da Política Nacional e Alimentação e Nutrição: 1. Organização da Atenção Nutricional; 2. Promoção da Alimentação Adequada e Saudável; 3. Vigilância Alimentar e Nutricional; 4. Gestão das Ações de Alimentação e Nutrição; 5. Participação e Controle Social; 6. Qualificação da Força de Trabalho; 7. Controle e Regulação dos Alimentos; 8. Pesquisa, Inovação e Conhecimento em Alimentação e Nutrição; 9. Cooperação e articulação para a Segurança Alimentar e Nutricional (Brasil, 2012). São atributos essenciais da APS o acesso de primeiro contato, longitudinalidade, coordenação e integralidade e atributos derivados a orientação familiar, comunitária e competência cultural (Starfield, 2002).

Para caracterização das ações, os dados foram resumidos e agrupados por macrorregião, tema da ação, tipo de abordagem (grupo ou individual), público-alvo, profissionais envolvidos, existência de articulação entre outros pontos de atenção ou parcerias intersetoriais, diretriz da PNAN e atributo da APS que mais se correlacionaram. A partir dessa categorização buscou-se identificar avanços e desafios atuais em relação à implementação das ações da PNAN de forma a contribuir com o fortalecimento da Atenção Primária.

Para descrever os termos mais frequentes presentes nas experiências utilizou-se a ferramenta "nuvem de palavras", utilizando o software atlas TI, que é capaz de identificar os termos mais frequentes de um texto e destacálos mediante a seleção automática do tamanho da fonte, em função da frequência, auxiliando, assim, na identificação de termos emergentes e contribuindo para desvendar as categorias empíricas.

\section{RESULTADOS}

Entre os trabalhos avaliados, conforme pode-se observar na Tabela 1, 41\% foi proveniente da região sudeste, seguida da região sul $(23,1 \%)$ e nordeste $(20,5 \%)$. Os atributos predominantes foram a orientação comunitária $(71,8 \%)$ e acesso de $1^{\circ}$ contato $(61,5 \%)$, sendo a obesidade o tema mais recorrente (61,5\%). A maioria das ações ocorreram em grupos de atendimento (51,3\%), tendo como principal público alvo, os adultos (59\%). Os nutricionistas participaram de $61,5 \%$ das ações, seguidos dos psicólogos (25,6\%), tendo sido mencionado articulação intersetorial em $38,5 \%$ das experiências apresentadas.

A análise das experiências mostrou que a maioria das ações foram desenvolvidas em Unidades Básicas de Saúde (UBS) da Atenção Primária com adultos com sobrepeso/obesidade. As principais intervenções realizadas pelas equipes de saúde com foco na prevenção e controle da obesidade abordaram a vigilância alimentar e nutricional (VAN) e/ou promoção da alimentação adequada e saudável (PAAS) e algumas incluíam além de 
VAN e PAAS, ações de promoção da atividade física e práticas integrativas e complementares (PICS).

Tabela 1 - Categorias de análises das experiências de Alimentação e Nutrição do Prêmio APS Forte, 2019.

\begin{tabular}{|c|c|c|}
\hline Categorias & n. & $\%$ \\
\hline \multicolumn{3}{|l|}{ Região } \\
\hline Sudeste & 16 & 41,0 \\
\hline Sul & 09 & 23,1 \\
\hline Nordeste & 8 & 20,5 \\
\hline Centro- Oeste & 03 & 7,7 \\
\hline Norte & 03 & 7,7 \\
\hline \multicolumn{3}{|l|}{ Diretrizes da PNAN } \\
\hline Promoção da Alimentação Adequada e Saudável & 31 & 79,5 \\
\hline Vigilância Alimentar e Nutricional & 17 & 43,6 \\
\hline Organização da Atenção Nutricional & 4 & 10,3 \\
\hline Articulação e cooperação para SAN & 3 & 7,7 \\
\hline Gestão das ações de Alimentação e Nutrição & 2 & 5,1 \\
\hline Controle e regulação dos alimentos & 1 & 2,6 \\
\hline Qualificação da força de trabalho & 1 & 2,6 \\
\hline \multicolumn{3}{|l|}{ Atributos da APS } \\
\hline Orientação Comunitária & 28 & 71,8 \\
\hline Acesso de 10 contato & 24 & 61,5 \\
\hline Longitudinalidade & 23 & 59,0 \\
\hline Orientação familiar & 11 & 28,2 \\
\hline Integralidade no cuidado & 6 & 15,4 \\
\hline Coordenação do cuidado & 6 & 15,4 \\
\hline \multicolumn{3}{|l|}{ Temática } \\
\hline Obesidade & 24 & 61,5 \\
\hline Ações de Promoção da Alimentação Adequada e Saudável & 5 & 12,8 \\
\hline Saúde na Escola & 4 & 10,3 \\
\hline Gestão das ações de Alimentação e Nutrição & 2 & 5,1 \\
\hline Atendimento às necessidades alimentares especiais & 1 & 2,6 \\
\hline Alimentação na saúde prisional & 1 & 2,6 \\
\hline Ações de Vigilância Alimentar e Nutricional & 1 & 2,6 \\
\hline Suplementação de micronutrientes & 1 & 2,6 \\
\hline \multicolumn{3}{|l|}{ Tipo de Atendimento } \\
\hline Grupos de atendimento & 20 & 51,3 \\
\hline Ações de educação em saúde & 13 & 33,3 \\
\hline Consulta individual & 4 & 10,3 \\
\hline Pesquisa-ação & 2 & 5,1 \\
\hline \multicolumn{3}{|l|}{ Público-alvo } \\
\hline Adultos & 23 & 59,0 \\
\hline Crianças & 16 & 41,0 \\
\hline
\end{tabular}




\begin{tabular}{lcc} 
Adolescentes & 2 & 5,1 \\
\hline Profissionais envolvidos & & \\
\hline Nutricionistas & 24 & 61,5 \\
Psicólogos & 10 & 25,6 \\
Educadores físicos & 8 & 20,5 \\
Equipe Saúde da Família & 7 & 17,9 \\
Médicos & 7 & 17,9 \\
Enfermeiros & 6 & 15,4 \\
Núcleo de Apoio à Saúde da Família & 5 & 12,8 \\
Assistentes Sociais & 4 & 10,3 \\
Fisioterapeutas & 3 & 7,7 \\
\hline Articulação & \multicolumn{3}{c}{38,5} \\
\hline Intersetorial & 15 & 3,6 \\
Intrassetorial & 1 & 2,6 \\
\hline
\end{tabular}

Na nuvem de palavras apresentada na figura 1 visualizam-se as palavras que apareceram com maior frequência, demonstrando que as atividades de alimentação e nutrição são vistas pelas equipes de APS como um potente recurso para promoção da saúde. Desta forma, denotam o reconhecimento da magnitude da obesidade pelas equipes de saúde no território adstrito, sendo enxergada, mesmo que de forma sutil, como um problema real que exige uma reorganização do processo de trabalho dos profissionais.

Figura 1 - Nuvem de palavras dos relatos de Alimentação e Nutrição do Prêmio APS Forte, 2019

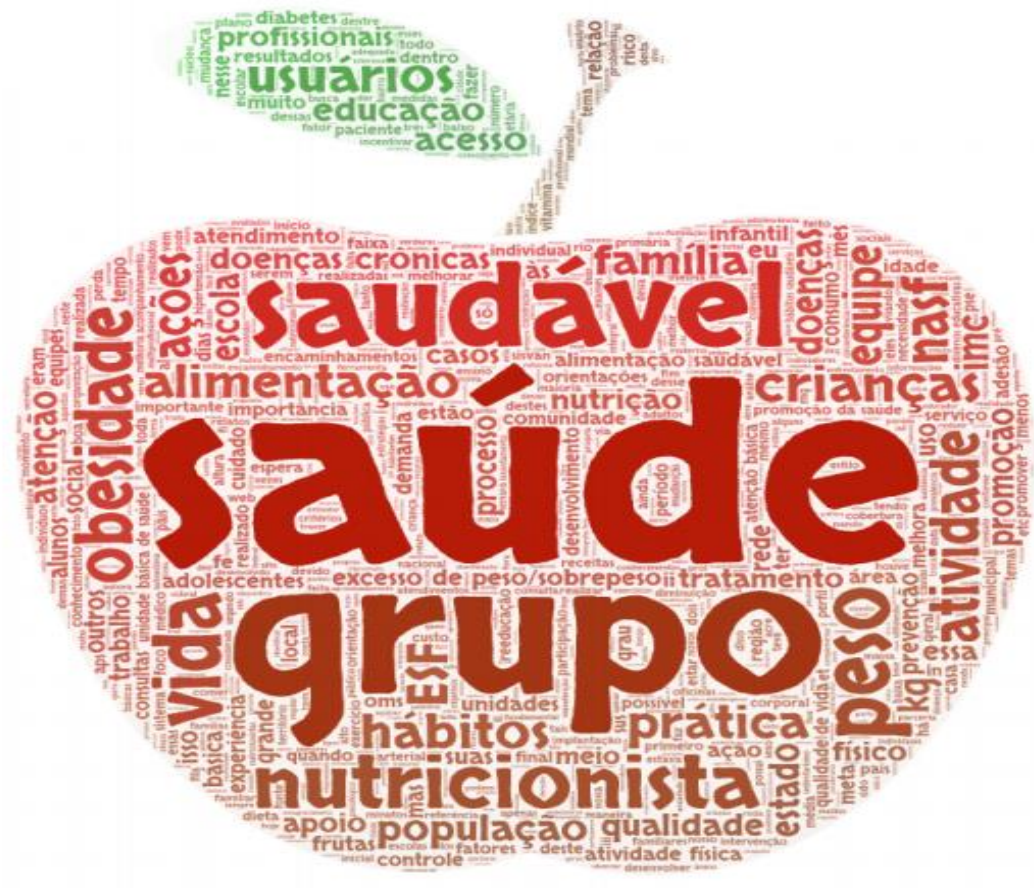


Também foi identificado experiências voltadas ao enfrentamento da obesidade infantil, sendo a maior parte das ações realizadas em parceria com as escolas. Destacam-se experiências que agregam a vigilância alimentar e nutricional, com foco na avaliação do estado nutricional de escolares, além de ações de promoção da alimentação saudável e prática de atividade física no ambiente escolar. Além de experiências que ofertaram ações de promoção e cuidado nas UBS para as crianças identificadas com obesidade. Algumas experiências destacaram a importância de usar metodologias adequadas para cada faixa etária das crianças e valorizar o lúdico nas ações.

A maioria das ações organizadas pelas equipes ocorreu por meio de grupos conduzidos por profissionais da equipe mínima da equipe de saúde da família (ESF), com grande frequência na participação de outros profissionais, fortalecendo a atuação multidisciplinar. E, os principais núcleos profissionais envolvidos nas ações foram os nutricionistas, psicólogos, educadores físicos e enfermeiros, sendo observado pouco envolvimento do profissional médico. As metodologias utilizadas variaram muito entre as experiências, porém, várias delas usaram metodologias ativas, participativas, centradas na pessoa e mais lúdicas, quando conduzidas com crianças. Foi frequente o uso do telefone celular e de ferramentas como o aplicativo de conversa instantânea para aumentar a motivação dos usuários em intervenções de seguimento.

Merece destaque a experiência "Comida de verdade na ESF, do município de Estância Velha/RS" que apresentou a integração entre grupos de promoção da alimentação adequada e saudável promovidos pela equipe de saúde com os produtores locais, possibilitando aos usuários acesso à alimentos in natura por meio de feiras, acompanhadas de orientações do Guia Alimentar para a população brasileira. Essa parceria fortalece as ações de promoção da saúde da saúde no território e contribuiu para o aumento do consumo de alimentos saudáveis pela população assistida. Também se destacaram como ofertas de intervenções diferenciadas as experiências que associaram alimentação saudável e PICS, como relatado em "Mineiros mais leves, do município de Mineiros/GO" e "AliMente, do município de Seara/SC", que utilizaram auriculoterapia para potencialização da perda de peso e redução de ansiedade.

A maioria dos relatos apresentou como resultado avaliações qualitativas feitas pelas próprias equipes, como: relatos de perda de peso, melhora da autoestima, aumento no aproveitamento integral dos alimentos; redução do perímetro da cintura, melhoria dos níveis de glicose, colesterol e triglicérides e dos padrões de consumo alimentar. O acompanhamento psicológico associado à orientação alimentar foi outro ponto importante destacado no cuidado com os pacientes, contribuindo para melhor autoestima, autocuidado e valorização de si mesmo.

Os relatos encaminhados foram de temáticas diversas e, apesar da maioria tratar de grupos, evidencia-se também experiências sobre o organização do acesso; suplementação de micronutrientes; estímulo ao consumo de frutas, legumes e verduras; elaboração de protocolos pela APS para outros níveis de atenção; além de estruturação da linha de cuidado de sobrepeso e obesidade no município, garantindo assim o fortalecimento de atributos da APS como a longitudinalidade, a integralidade e coordenação do cuidado. Por fim, ressalta-se experiência realizada integrando a APS à saúde prisional que propiciou maior segurança sanitária dos alimentos consumidos pelos indivíduos privados de liberdade, resultando na diminuição da incidência de infecções gastrointestinais.

A análise das experiências em relação às diretrizes da PNAN mostrou que a maioria dos trabalhos relacionou-se com a promoção da alimentação adequada e saudável, a vigilância alimentar e nutricional, a organização da atenção nutricional, aparecendo por vezes, de forma concomitante, mas também verificou-se experiências que se enquadram nas diretrizes de articulação e cooperação para a segurança alimentar e nutricional, gestão das ações de 
alimentação e nutrição e qualificação da força de trabalho, em menor proporção.

Entre os atributos da APS, foi possível observar que as experiências fortalecem o primeiro contato (exemplo a identificação dos obesos) e o cuidado longitudinal (intervenções de longa duração) com forte componente de orientação comunitária. Também foram identificadas experiências que fortalecem a integralidade e a coordenação do cuidado a partir da APS, com a organização da demanda, os fluxos de encaminhamento e protocolos, visto que o cuidado de indivíduos obesos, uma vez estratificado seu risco, demandará intervenções da atenção especializada.

\section{DISCUSSÃO}

De acordo com as análises das experiências recebidas, observou-se uma predominância no reconhecimento da obesidade por parte das equipes de saúde como um problema relacionado à má alimentação e que demanda um esforço interdisciplinar para obter melhores resultados no âmbito da Atenção Primária à Saúde. Porém, recente publicação que avaliou motivos de consulta na APS brasileira no período de setembro de 2018 a agosto de 2019 mostrou que $26,38 \%$ das condições/problemas avaliados correspondiam à hipertensão arterial, 10,51\% ao diabetes e apenas, 2,85\% correspondiam à obesidade, apontando para uma possível invisibilidade da obesidade na rotina das equipes (Bortolini et al., 2020).

De acordo com inquéritos nacionais, podemos inferir que a cada 100 adultos que entram em uma UBS, 50 apresentam excesso de peso, chegando a 60 em algumas capitais do Brasil (Brasil, 2020). Dessa forma, considerando que a APS possui capacidade para resolver $80 \%$ dos problemas de saúde de uma determinada população (Starfield, 2002), essa deve ser o lócus prioritário para prevenção e controle da obesidade no Brasil, enfatizando-se a necessidade de fortalecimento do acesso nesse nível de atenção para atendimento dessa condição, conciliando ações de identificação, assistência, prevenção e promoção da saúde, além de coordenar a atenção prestada nos outros níveis do sistema, garantindo a integralidade do cuidado.

Evidências recentes sugerem que os melhores resultados de perda de peso são obtidos com abordagens multidisciplinares (Bright et al., 2019); estruturadas, facilmente adaptáveis para cada indivíduo, disponível em locais diferentes, que incorporem toda a família e promovam mudanças sustentáveis no estilo de vida (Kennedy et al., 2016); Para maximizar a efetividade do manejo de peso, recomenda-se a aplicação de comunicação centrada no paciente e decisão compartilhada a respeito das metas de peso (Turer, 2015); As orientações dietéticas devem ser consistentes e claras para as mudanças específicas na dieta, como alcançá-las e como monitorá-las (Phillips et al., 2012); As intervenções sobre obesidade devem ser breves, direcionadas e efetivas (Fitzpatrick et al., 2016) e envolver a abordagem de questões psicossociais ou comorbidades psiquiátricas associadas ao fracasso no tratamento da obesidade, uma vez que esse pode ser um fator limitante no tratamento da síndrome metabólica (Ferrari et al., 2017).

O cuidado das pessoas com obesidade na APS, deve considerar como objetivo a perda de peso, mas também a estabilização da condição crônica, nem sempre sendo possível reduzir o IMC para menos de 25 . A perda de $5 \%$ a $10 \%$ do peso original por um ou mais anos está associada a melhorias nos principais fatores de risco e comorbidades de doenças crônicas não transmissíveis, como a redução da pressão arterial (American College of Cardiology/American Heart Association, 2014; Wing et al., 2011), risco cardiovascular (Naslund et al., 2016), e melhoria da tolerância à glicose (Erlandson, Ivey e Seikel, 2016).

Recente publicação apontou que entre os principais obstáculos para que o usuário obeso busque o serviço de saúde está o estigma e o preconceito vivenciado (Bortolini et al., 2020). É importante que estes sejam tratados como demais portadores de outras doenças crônicas sem culpabilização. 
As experiências do prêmio APS forte, desenvolvidas pelas equipes de saúde da família, mostraram que estas se preocupam em desenvolver ações para prevenção e controle da obesidade infantil e, demonstraram o quanto a APS pode contribuir com mudanças positivas nas práticas alimentares e compreensão sobre alimentação saudável das crianças envolvidas. esse reconhecimento é importante para prevenir obesidade em todos ciclos de vida, pois uma criança e adolescente com obesidade apresentam cerca de cinco vezes mais chances de serem obesos na idade adulta (Llewellyn et al., 2016).

As ações que podem ser realizadas na APS e que mais contribuem com a prevenção da obesidade infantil são: orientação da alimentação da gestante e controle do ganho de peso gestacional; promoção do aleitamento materno e da introdução da alimentação complementar saudável; utilização de metodologias centradas nas pessoas para estimular hábitos saudáveis e organização de ações intersetoriais na escola ou no território (WHO, 2016). As ações desenvolvidas para prevenção do sobrepeso e obesidade no âmbito escolar entre os relatos analisados, encontravam-se em consonância com as propostas pelo Programa Crescer Saudável, que tem foco a vigilância, promoção e cuidado, atualmente sendo implementado em mais de 4 mil cidades no país.

As experiências ressaltaram a multiprofissionalidade como uma prática para aumento do escopo de ações ofertadas (Salvador, 2011), refletindo sobre a melhoria na qualidade de vida dos usuários e de seus familiares e na resolutividade da APS, tendo em vista que interfere diretamente sobre o atributo de integralidade (Brandão, 2020). Poucas experiências tiveram a participação de médicos de família, porém o envolvimento desse profissional em intervenções voltadas ao enfrentamento de obesidade infantil, têm se mostrado efetivas (Bhuyan et al., 2015). A atuação multiprofissional permite que os pacientes ampliem a compreensão sobre sua condição, uma vez que profissionais não médicos geralmente transmitem as mesmas informações em diferentes maneiras, facilitando a compreensão dos pacientes sobre a importância da adesão ao tratamento da doença (Cristina et al., 2017).

A protagonização das ações por profissionais de núcleos de saberes distintos da equipe mínima, remete à reflexão de que essas representam a minoria das equipes de atenção primária (Pires, 2017), cuja composição conta com o médico, enfermeiro, técnico em enfermagem, podendo ter ou não, agente comunitário de saúde. Os enfermeiros, em geral, apresentam maior envolvimento com as ações de promoção da saúde, principalmente relacionadas com doenças crônicas não transmissíveis, no entanto, necessitam aprimorar suas habilidades metodológicas na realização de atividades de educação em saúde (Duranza, 2010), e capacidade de atuação em ações de prevenção primária e promoção da saúde (Paraizo, 2018).

Estudo qualitativo realizado no município de Santa Cruz do Sul - RS, que teve como objetivo analisar os processos de trabalho do NASF-AB e matriciamento por meio da visão dos profissionais das equipes de Saúde da Família, que envolveu médicos, enfermeiros, técnicos de enfermagem, dentistas e agentes comunitários de saúde, verificou que os vínculos construídos entre as equipes facilitavam o trabalho nos territórios, mas não eram de consenso de todos os profissionais. Os processos de trabalho ainda não eram claros a todos, pois alguns profissionais não participavam do trabalho conjunto e discussão de casos entre as equipes, indicando ainda o desejo de realizar o encaminhamento e transferir o cuidado a outro profissional em vez de atuar de forma conjunta (Morinel, 2019).

Em Santa Catarina, estudo realizado com gestores e profissionais do Núcleo de Apoio à Saúde da Família, encontrou que essas equipes, no contexto do matriciamento, se devidamente articuladas com a APS, promoviam, apoiavam e fortaleciam a atuação dos generalistas da ESF e também apareciam como especialistas que atuavam no seu núcleo de saber e auxiliavam as equipes de ESF no ordenamento e coordenação do cuidado, podendo contribuir, assim, em parte, para diminuir os estrangulamentos da 
APS. Enquanto ressalva, apontou que o NASF deveria ser uma estratégia claramente delineada em relação aos seus propósitos, diretrizes e possibilidades estruturais e de atuação, sob o risco de funcionar como uma equipe sem identidade clara no contexto da RAS, com pouca efetividade e sustentabilidade (Souza e Medina, 2018).

A APS é apontada como o nível de atenção ideal para promover mudanças de estilo de vida/manejo de fatores de risco. Contudo, a capacidade de fornecer intervenções com intensidade suficiente é uma importante précondição para alcançar este objetivo, requerendo-se que os profissionais que atuam nesse nível de atenção se sintam aptos a fornecer intervenções de maior duração e frequência (Dennis et al., 2012) et al., 2012). Por ser a porta de entrada dos usuários no serviço, a APS tem suma importância na identificação dos indivíduos com obesidade, bem como, no auxílio do início do tratamento, procedendo os encaminhamentos necessários e mantendo a longitudinalidade no cuidado com os demais serviços da rede (Soeiro et al., 2019)

A realização de grupos de educação em saúde realizados por profissionais da APS tem demonstrado resultados positivos sobre comportamentos alimentares de adultos, sendo capaz de promover melhorias significativas no aumento do consumo diário de frutas, vegetais, pão rico em fibras e peixe (Mitchell et al., 2017). Os grupos para indivíduos com obesidade, quando acontecem com a frequência necessária, contribuem para a perda de peso gradativa, redução da circunferência abdominal e melhoria de hábitos saudáveis (Doria, 2013; Soeiro et al., 2019). Além disso, mostram-se eficazes por permitir uma maior interação social e apoio mútuo entre os participantes, promovendo maior adesão ao processo de emagrecimento e fortalecimento de vínculo com a equipe de saúde (Doria, 2013). Para potencializar as práticas de promoção da saúde realizadas por profissionais da Estratégia de Saúde da Família, visando o controle do excesso de peso, recomenda-se ainda que possam ser criadas condições para que os discursos acerca dos cuidados com o corpo sejam discutidos e questionados; e que, além disso, essas práticas possam trazer subsídios para que os cuidados com a alimentação, o engajamento em atividades físicas, dentre outras atitudes de mudança de hábitos, sejam vivenciadas como atividades de múltiplas potências e não somente como um esforço de adaptação à norma estética/médica do corpo magro/saudável (Camargo, 2015).

A saúde de um indivíduo ou uma população é determinada por fatores individuais, mas é fortemente influenciada pelo ambiente social e físico, por comportamentos que são culturais ou socialmente determinados e pela natureza da atenção à saúde ofertada (Starfield, 2002). Cada vez mais, tem-se assumido que a obesidade não é apenas uma questão individual e biológica, mas sim, que possui determinação social e dependente dos ambientes onde as pessoas estão inseridas, como principais determinantes (WHO, 2016)).

A Política Nacional de Promoção da Saúde considera que os sujeitos e coletividades são produtos da influência exercida pelos ambientes de vida e trabalho em que se situam, bem como o aumento da capacidade para tomada de decisão se dar por meio do desenvolvimento de habilidades pessoais e competências que os empoderem. Em relação às atividades de promoção à saúde descritas nos relatos, identificou-se como um desafio a boa capacidade de articulação com outros setores para a implementação de medidas mais abrangentes que interferissem sobre os determinantes e ambientes alimentares dos usuários. Observou-se que as ações ofertadas, geralmente eram focadas apenas nos indivíduos por sua condição e na mudança de seu estilo de vida.

Quanto à convergência com os atributos da APS, as intervenções relatadas dialogam majoritariamente com os atributos de orientação comunitária, acesso de primeiro contato e longitudinalidade. Assim, percebe-se a necessidade das equipes da APS reconhecerem à magnitude e relevância dos agravos associados à má alimentação na comunidade e esforçar-se em acolhê-las, estruturando ações e 
programas que possam prestar a assistência ao longo do tempo para intervir sobre essas condições.

Destaca-se também a presença de experiências alinhadas aos atributos de orientação familiar e integralidade, que por sua vez, associa-se à valorização das condições econômicas, sociais e culturais das famílias adstritas, além de remeter à intersetorialidade, imprescindível para a garantia do Direito Humano à Alimentação Adequada e a Segurança Alimentar e Nutricional da população brasileira. Também foram identificados trabalhos com interface à coordenação do cuidado, tratando-se de estratégias de organização da demanda, bem como, de estabelecimento de fluxos e protocolos de encaminhamentos para especialistas disponíveis na APS e demais níveis de atenção.

Considerando que a obesidade é uma condição que impacta negativamente da saúde da população, pode-se elencar como estratégias primordiais para a melhor efetividade das intervenções/atividade de alimentação e nutrição na APS: disponibilizar protocolos de cuidado que possam legitimar a realização de condutas efetivas; qualificar habilidades dos profissionais em relação à comunicação e tecnologias do cuidado; disponibilizar ferramentas de tele consultoria e realizar formações que incluam além do diagnóstico e tratamento, ações que podem ser realizadas no âmbito da APS para fortalecimento de todos os seus atributos e a intersetorialidade.

\section{CONCLUSÃO}

No Brasil, a atenção primária tem se mostrado imprescindível para implementação e a efetivação dos princípios do SUS, tendo alcançado cobertura de mais de $50 \%$ da população brasileira, reduzindo a mortalidade infantil e as internações por condições sensíveis, ampliando o acesso a serviços de saúde para populações vulneráveis. Entretanto, permanece uma disparidade na qualidade da atenção ofertada, persistindo ainda o desafio de ampliar o acesso e a capacidade de resposta frente a novos e antigos agravos da saúde no Brasil (OPAS, 2018).

No âmbito da alimentação e nutrição, observase que ainda há um longo caminho a percorrer, especialmente no tocante à prevenção de doenças crônicas como sobrepeso e obesidade em todas as faixas etárias relacionadas à alimentação inadequada. De maneira geral, os profissionais reconhecem o problema da obesidade, no entanto, a maioria das intervenções focam apenas ações de educação em saúde, sendo a obesidade um tema que demanda fortalecimento de todos os atributos da APS e articulação intersetorial, principalmente entre saúde, educação, assistência social e agricultura familiar. 


\section{NOTAS E REFERÊNCIAS}

AMERICAN COLLEGE OF CARDIOLOGY/AMERICAN HEART ASSOCIATION. Expert panel report: Guidelines (2013) for the management of overweight and obesity in adultsObesity, 2014.

ARAÚJO, L. U. A. DE et al. Avaliação da qualidade da atenção primária à saúde sob a perspectiva do idoso. Ciencia e Saude Coletiva, 2014.

BHUYAN, S. S. et al. Integration of public health and primary care: A systematic review of the current literature in primary care physician mediated childhood obesity interventionsObesity Research and Clinical Practice, 2015.

BORTOLINI, G. A. et al. Ações de alimentação e nutrição na atenção primária à saúde no Brasil. p. 1-8, 2020.

BRANDÃO, J. R. DE M. O(s) Sistema(s) Primário(s) de Saúde Canadense sob uma perspectiva brasileira: discutindo os Atributos Starfield. Ciencia \& saude coletiva, v. 25, n. 4, p. 1413-1420, 2020.

BRASIL. MINISTRO DE ESTADO DA SAÚDE. Portaria MS n 710, de 10 de junho de 1999. Aprova a Política Nacional de Alimentação e Nutrição. [s.I: s.n.].

Pesquisa de Orçamentos Familiares: 2008-2009. Antropometria e Estado Nutricional de Crianças, Adolescentes e Adultos no Brasil. Biblioteca do Ministerio do Planejamento, Orçamento e Gestão, 2010.

. Política Nacional de Alimentaçao e Nutrição (PNAN). [s.I: s.n.].

BRASIL, I. Pesquisa de orçamentos familiares 2017-2018 : avaliação nutricional da disponibilidade domiciliar de alimentos no Brasil. Rio de Janeiro: [s.n.].

BRIGHT, D. et al. Tipping the scales: Provider perspectives on a multi-disciplinary approach to obesityExperimental Biology and Medicine, 2019.

CAMARGO, T. S. Sempre Alerta! Uma investigação acerca da (auto)vigilância e a gestão de si em um grupo de emagrecimento. TEXTURA - Revista de Educação e Letras, 2015.

CARVALHO, E. A. DE A. et al. Obesity: epidemiological aspects and prevention. Revista Médica de Minas Gerais, v. 23, n. 1, p. 74-82, 2013.

CASTRO, R. C. L. DE et al. Avaliação da qualidade da atenção primária pelos profi ssionais de saúde: Comparação entre diferentes tipos de serviços. Cadernos de Saude Publica, 2012.

CRISTINA, K. et al. Adherence to nutritional orientations : a literature review Adesão às orientações nutricionais : uma revisão de literatura. v. 12, n. 1, p. 249-274, 2017.

DABAS, A.; SETH, A. Prevention and Management of Childhood Obesity. Indian journal of pediatrics, v. 85, n. 7, p. 546-553, 1 jul. 2018.

DENNIS, S. et al. Which providers can bridge the health literacy gap in lifestyle risk factor modification education: A systematic review and narrative synthesisBMC Family Practice, 2012.

DORIA, R. L. Grupo de apoio a pessoa com obesidade na Atenção BásicaBrusque-SC: An Congr Bras Med Fam Comunidade., 2013

DURANZA, R. L. C. A produção de cuidado para os portadores de diabetes mellitus tipo 2 na atenção primária de saúde. Usp, 2010. 
ERLANDSON, M.; IVEY, L. C.; SEIKEL, K. Update on office-based strategies for the management of obesity. American Family Physician, v. 94, n. 5, p. 361-368, 2016.

FACCHINI, L. A.; TOMASI, E.; DILÉLIO, A. S. Qualidade da Atenção Primária à Saúde no Brasil: avanços, desafios e perspectivas. Saúde em Debate, 2018.

FERRARI, G. D. et al. A multidisciplinary weight-loss program: The importance of psychological group therapy. Motriz. Revista de Educacao Fisica, v. 23, n. 1, p. 47-52, 1 jan. 2017.

FITZPATRICK, S. L. et al. An Evidence-based Guide for Obesity Treatment in Primary CareAmerican Journal of Medicine, 2016.

GERMAN, A. J. Obesity Prevention and Weight Maintenance After Loss. The Veterinary clinics of North America. Small animal practice, v. 46, n. 5, p. 913-29, 1 set. 2016.

KEMPER, E. S. Avaliação da qualidade da Atenção Primária no Programa Mais Médicos. APS EM REVISTA, 2019.

KENNEDY, B. M. et al. Perceptions of obesity treatment options among healthcare providers and lowincome primary care patients. Ochsner Journal, 2016.

LANGER, O. Prevention of obesity and diabetes in pregnancy: is it an impossible dream? American journal of obstetrics and gynecology, v. 218, n. 6, p. 581-589, 1 jun. 2018.

LLEWELLYN, A. et al. Childhood obesity as a predictor of morbidity in adulthood: A systematic review and meta-analysis. Obesity Reviews, 2016.

MALTA, D. C. et al. Mortalidade por doenças crônicas não transmissíveis no Brasil e suas regiões, 2000 a 2011. Epidemiologia e Serviços de Saúde, 2014.

Fatores de risco relacionados à carga global de doença do Brasil e Unidades Federadas, 2015. Revista Brasileira de Epidemiologia, 2017.

MITCHELL, L. J. et al. Effectiveness of Dietetic Consultations in Primary Health Care: A Systematic Review of Randomized Controlled Trials. Journal of the Academy of Nutrition and Dietetics, 2017.

MORINEL, I. C. O papel do Núcleo Ampliado de Saúde da Famíia e Atenção Básica (NASF-AB). [s.I.] UFRGS, 2019.

NASLUND, J. A. et al. Lifestyle Intervention for People with Severe Obesity and Serious Mental Illness. American Journal of Preventive Medicine, 2016.

NILSON, E. A. F. et al. Custos atribuíveis a obesidade, hipertensão e diabetes no Sistema Único de Saúde, Brasil, 2018. Revista Panamericana de Salud Pública, v. 44, p. 1, 10 abr. 2020.

OPAS. 30 anos de SUS: OPAS/OMS lança publicação sobre experiências acumuladas em sua história de cooperação técnica com Brasil.

PARAIZO, C. M. S. Significado do cuidado à pessoa com diabetes mellitus na perspectiva de enfermeiros da atenção básica de saúde. [s.I.] Universidade Federal de Alfenas, 2018.

PHILLIPS, K. et al. Counselling patients about behaviour change: The challenge of talking about diet. British Journal of General Practice, 2012.

PIRES, A. C. L. Brasília 2017. Dissertação (Mestrado em Saúde Coletiva) - Universidade de Brasília, p. 134, 
2017.

SALVADOR, A. DE S. Construindo a Multiprofissionalidade: um Olhar sobre a Residência Multiprofissional em Saúde da Família e Comunidade. Revista Brasileira de Ciências da Saúde, 2011.

SOEIRO, R. L. et al. Educação em Saúde em Grupo no Tratamento de Obesos Grau III: um Desafio para os Profissionais de Saúde. Revista Brasileira de Educação Médica, v. 43, n. 1 suppl 1, p. 681-691, 2019.

SOUZA, T. S.; MEDINA, M. G. Nasf: fragmentação ou integração do trabalho em saúde na APS? TT - Nasf: fragmentation or integration of health work in PHC? Saúde debate, 2018.

STARFIELD, B. Atenção Primária - Equilíbrio entre necessidades de saúde, serviços e tecnologia. Brasília: UNESCO, Ministério da Saúde, 2002.

SWINBURN, B. A. et al. The Global Syndemic of Obesity, Undernutrition, and Climate Change: The Lancet Commission reportThe Lancet, 2019.

TREMMEL, M. et al. Economic burden of obesity: A systematic literature reviewinternational Journal of Environmental Research and Public Health, 2017.

TURER, C. B. Tools for successful weight management in primary care. American Journal of the Medical Sciences, 2015.

WHO. Ending Childhood Obesity Report. Geneva: Wold Health Organization, 2016.

WING, R. R. et al. Benefits of modest weight loss in improving cardiovascular risk factors in overweight and obese individuals with type 2 diabetes. Diabetes Care, 2011. 